\title{
How Group Cohesion Promotes the Emergence of Cooperation in Public Goods Game Under Conditional Dissociation
}

\author{
Qu Xinglong ${ }^{1}$, Cao Zhingang ${ }^{2}$, Yang Xiaoguang ${ }^{3,4}$, The Anh Han ${ }^{5}$ \\ ${ }^{1}$ Research Center of Information Technology \& Social and Economic Development, Hangzhou Dianzi Univer- \\ sity, Xiasha Higher Education Zone, Hangzhou, Zhejiang Province 310018, China \\ ${ }^{2}$ Department of Economics, School of Economics and Management, Beijing Jiaotong University, 3 \\ Shangyuancun Haidian District, Beijing 100044, China \\ ${ }^{3}$ Academy of Mathematics and Systems Science, Chinese Academy of Sciences, No.55 Zhong Guan Cun \\ Donglu, Beijing 100190, China \\ ${ }^{4}$ University of Chinese Academy of Sciences, No.19(A) Yuquan Road, Shijingshan District, Beijing 100049, \\ China \\ ${ }^{5}$ School of Computing, Media, and Arts, Teesside University, Middlesbrough Tees Valley TS1 3BX, United King- \\ dom \\ Correspondence should be addressed to zhigangcao@amss.ac.cn
}

Journal of Artificial Societies and Social Simulation 22(3) 5, 2019

Doi: 10.18564/jasss.4070 Url: http://jasss.soc.surrey.ac.uk/22/3/5.html

Received: 31-01-2019

Accepted: 27-05-2019

Published: 30-06-2019

\begin{abstract}
Leaving is usually an option for individuals if they cannot tolerate their defective partners. In a twoplayer game, when a player chooses to leave, both she and her opponent become single players. However, in a multi-player game, the same decision may have different consequences depending on whether group cohesion exists. Players who choose not to leave would still be united together rather than be separated into singletons if there is cohesion among them. Considering this difference, we study two leaving mechanisms in public goods games. In the first mechanism, every player would be single once any of the group members leaves. In the second, we assume group cohesion exists that members who don't leave form a union. In our model, each player adopts a trigger strategy characterized by a threshold: she leaves if the number of defectors in her group exceeds the threshold. We find that under both mechanisms, when the expected lifespan of individuals is long enough, cooperators with zero tolerance toward defection succeed in the evolution. Moreover, when cohesion exists in groups, cooperation is better promoted because the cooperators have a higher chance to play together. That is, group cohesion facilitates positive assortment and therefore promotes cooperation.
\end{abstract}

Keywords: Group Cohesion, Public Goods Game, Cooperation Emergence, Conditional Dissociation, Positive Assortment

\section{Introduction}

1.1 Cooperation is indispensable for a society to form and persist. However, cheaters may earn higher fitness and beat the cooperators in the evolution, and hence cooperation seems impossible in many scenarios resembling Prisoner's Dilemma. To explain the ubiquitousness of cooperation and the existence of society, several mechanisms promoting cooperation have been proposed and studied in-depth (Nowak 2006; Perc et al. 2017). These mechanisms usually share a common character, which is termed as positive assortment (Doebeli \& Hauert2005, Eshel \& Cavalli-Sforza 1982, Fletcher \& Doebeli|2006, 2009) that allows cooperators to preferentially interact among themselves.

1.2 Moreover, it has been shown that when players are given the option to leave their current partners and regroup with others, cooperation is also promoted (Aktipis 2004, Fujiwara-Greve \& Okuno-Fujiwara|2009; Izquierdo et al. 2014, 2010. Joyce et al.|2006: Qu et al.|2016 Schuessler 1989||Vainstein et al.|2007). This topic is of interest to researchers in different fields (Fujiwara-Greve \& Okuno-Fujiwara 2009), because it is easy to think of various examples where people dissolve different kinds of relationships: workers resign from companies and find new jobs; people migrate from one country to another; and couples may divorce and re-marry. 
1.3 Most of the previous studies concerning this topic focus on the Prisoner's Dilemma game. Since this is a twoplayer game, it is unsuitable for situations where people form multilateral relationships. In bilateral relationships, if one individual leaves, it is natural to assume that the other individual would be single. However, it may not be the case for multilateral relationships. For example, many music bands including The Beatles, Pink Floyd, and Xiao Hu Dui etc., break up completely after a single member's leaving. On the other hand, just like the resignation and migration issues we just mentioned above, companies or countries seldom collapse because of a few individuals' leaving. Except for the group sizes, a key difference lies in whether cohesion exists or not.

1.4 Cohesiveness is an important element that influences a group's performance. It is studied extensively in various domains, including psychology, organization behavior, sport, and education, etc. (Evans \& Dion 1991; Mullen \& Copper 1995). In his pioneering work, Festinger (1950) defines group cohesiveness as "the resultant forces which are acting on the members to stay in a group". This definition has been accepted by most subsequent researchers Mullen \& Copper 1995 notwithstanding some further refinements and modifications were proposed Carron \& Brawley 2000). In general, it is believed that high levels of group cohesion are advantageous or associated with better group performance (Gully et al. 1995). However, some empirical observations of the relation turn out to be negative (Høigaard et al.2006). This contradiction arises because we are not clear about how cohesiveness affects performance (Paskevich et al. 1999). To better understand this question from a micro perspective, we apply evolutionary game theory to study the impact of group cohesion on cooperation.

1.5 We extend the conditional dissociation model (Izquierdo et al. 2014, 2010, Qu et al. 2016) to public goods game and investigate two dissociation mechanisms corresponding to the above scenarios. Under the first mechanism, there is no cohesion. For a group of players, everyone would be single and enter the matching pool if anyone in the group dies or chooses to leave. So all players are single in the matching pool, and they would be rematched randomly to continue the game. Under the second mechanism, we assume the existence of cohesion. For a group of players, while those who leave would be single, the remaining ones would be united together rather than be single. So some players are single and the others are in unions in the matching pool. The rematching process is conducted in the following way. Given their cohesion, single players would first join existing unions (wherein the number of single players is the difference of the group size and the union size). The remaining single players are then randomly regrouped. It is worth pointing out that since all the unions can always be filled with single members, they would not be regrouped with other unions.

1.6 For each player, since leaving is also an option, her strategy should include at least two parts: the cooperation part and the leaving part. Despite the dissociation and rematching processes are different under the two mechanisms, players' strategy sets are the same in our model. For each individual, the cooperation part is fixed, i.e., she either always cooperates or always defects, regardless of histories and opponents she encounters. The leaving part is a trigger strategy that is characterized by a threshold. When the number of defectors in her group exceeds the threshold, she leaves. So the threshold represents a player's tolerance level towards defectors. In the Prisoner's Dilemma models of dissociation, it is unsuitable to define this tolerance level since there is at most one defective co-player. Thus, our multiplayer-game model also facilitates the investigation of the coevolution of cooperation and tolerance.

1.7 We have two main findings. First, when the expected lifespan of the individuals is long enough, cooperators are more likely to dominate the population under both dissociation mechanisms. This result is similar to those obtained from the Prisoner's Dilemma game models (Izquierdo et al.|2014, 2010, Qu et al.|2016). Our results reveal that when leaving is available, the winning cooperators always have zero tolerance toward defectors, which is significantly different from the previous conclusion that moderate tolerance benefits cooperation (Chen et al. 2009 Szolnoki \& Chen 2015). In these studies, some more sophisticated strategies and population structures are introduced. The tolerance level of a player not only affects her own choice of strategies but also the structure of the underlining populations. As a consequence, the systems meet a highest assortativity coefficient with moderate tolerance level.

1.8 Second, cooperation arises more quickly and in larger-scale when group cohesion exists. Roughly speaking, this result can also be attributed to the positive assortment effect. Since the winning cooperators are always intolerant, comparing how these two mechanisms promote cooperation is equivalent to comparing how intolerant cooperators behave under these two mechanisms. For the same strategy distributions, there should be an equal number of players in the matching pool under both mechanisms. In particular, the number of intolerant cooperators in the matching pool, all of whom are single, is almost equal under both mechanisms. Since some players are in unions under mechanism II, there are less single players in the matching pool. With equal intolerant cooperators but less single players, the probability that an intolerant cooperator meets another intolerant cooperator is larger under mechanism II, implying that the positive assortment effect is more significant. 


\section{Literature review}

1.9 Our research is mostly related to the study of conditional dissociation mechanisms. We use "conditional dissociation" to emphasize that players' leaving decisions are conditional on their opponents' actions. Other similar terms include exit (Schuessler 1989), walk away (Aktipis 2004), mobility (Vainstein et al. 2007), voluntarily separable (Fujiwara-Greve \& Okuno-Fujiwara 2009), out-for-tat (Hayashi|1993), migration (Li \& Ye 2018), and partner switch (Wubs et al. 2016) etc.

1.10 In some studies, individuals are assumed to be located on spatial lattices of periodic boundaries (Aktipis 2004; Chen et al. 2015, Li \& Ye 2018, Vainstein et al. 2007). Different migration rules have been investigated. For example, the individuals with walk-away strategy always move with random directions when encountering defectors (Aktipis 2004); the success-driven individuals move towards higher payoff locations (Helbing \& Yu 2009); aspiration-driven individuals move to neighbors that bring them greater satisfaction (Chen et al.2015) and the always-move individuals leave their current locations definitely and aimlessly.

1.11 As have been shown in many works, population structures have a significant impact on the evolution of cooperation (Rong et al. 2013, Szabó \& Fath 2007, Van Veelen et al. 2012). To better understand how dissociation promotes cooperation, some works consider well-mixed populations in order to rule out the effect of population structures (Fujiwara-Greve \& Okuno-Fujiwara 2009; | Izquierdo et al. 2014, 2010; Qu et al. 2016). In these works, when a player leaves her current opponent, both of them would enter a common matching pool. All the players in the matching pool would be randomly matched to continue interacting with others and receiving payoffs. These works find that the intolerant cooperative strategy is successful when players' expected lifespan is long enough.

1.12 Previous works on dissociation mostly focus on two-player models based on the Prisoner's Dilemma game and relatively few works consider public goods multi-player interactions. In the works of Hauert et al. (2002); Sasaki et al. (2007); Semmann et al. (2003), it is not everybody's obligation to participate in a game. Besides cooperating and defecting, the so-called loner strategy is introduced. In each round of interactions, loner players do not play with anyone but leave the game and receive a fixed baseline payoff (which is larger than zero but smaller than the fully cooperative payoff). Despite the fact that the participation in an interaction is optional, this dissociation is unconditional, i.e., players have to leave their current opponents after each interaction.

1.13 The rest of this paper is organized as follows. We describe the model in Section 2 present the simulation results in Section 3. provide the analytical results in Section 4 and then we conclude this paper in Section 5 with some further discussions.

\section{The Model}

2.1 We consider a population of $N$ individuals who are grouped randomly to play public goods games. Each group consists of $G$ players $(G<N$ and $G$ can divide $N)$. At the beginning of the game, each individual is endowed with one unit of wealth, and she has two options, either to cooperate $(C)$ or to defect $(D)$. A cooperator contributes one unit of her private wealth to the public pool, while a defector does not contribute. All the wealth in the public pool is multiplied by a multiplication factor $r(1<r<G)$ and then equally shared by all the players in that group. Let $a_{i}$ denote player $i$ 's action in a single game: $a_{i}=1$ if she cooperates, and $a_{i}=0$ otherwise. In summary, in a single round of the game, the payoff for player $i$ is

$$
u_{i}=\frac{r}{G} \cdot \Sigma_{j=1}^{G} a_{j}+\left(1-a_{i}\right) .
$$

It is easy to see that defection is a dominant strategy. Therefore, all players defect is the only Nash equilibrium and the only NSS (Neutrally Stable Strategy, which is a refinement of Nash equilibrium). Nevertheless, this is unsatisfactory, because all players cooperate is the desirable outcome.

2.2 Fortunately, if the public goods game is repeated infinitely, then cooperation is reachable. This classical result is usually referred to as the folk theorem (Fudenberg \& Maskin 1986; Rubinstein 1979). In the classical framework, the termination of the repeated games is exogenously determined. In this work, we study the conditional dissociation mechanism, under which players are allowed to leave their current opponents so the repeated games among them is terminated (Izquierdo et al.|2014, 2010, Qu et al.|2016. Once a player chooses to leave, she and her opponents enter the matching pool. All players in the matching pool will be rematched randomly to continue the game, until someone chooses to leave. This process is infinitely repeated. It is assumed that no player chooses to leave if all her opponents play $C$ in the current round. Therefore, it is possible that a group of players consecutively play for infinitely many rounds. However, if anyone plays $D$, then the group may dismiss. 


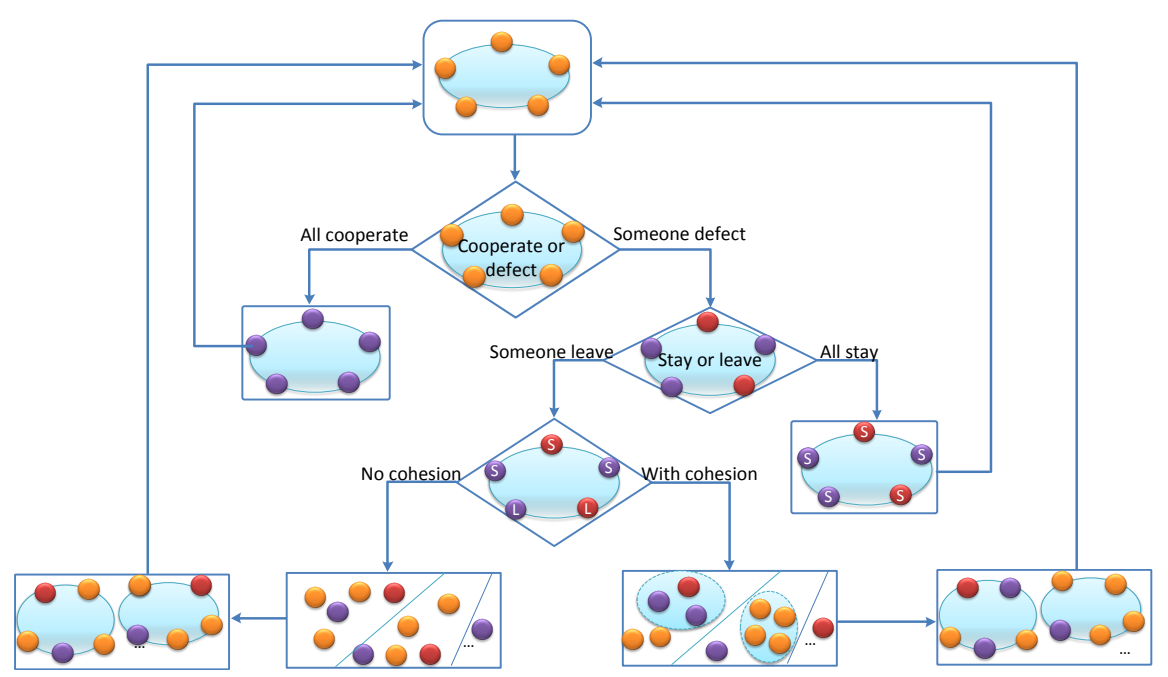

Figure 1: An illustration of the two dissociation mechanisms.

2.3 In previous works, the game studied is the Prisoner's Dilemma game Izquierdo et al.|2014,2010, Qu et al.|2016). Since it is a two-person game, a single player's leaving naturally leads both players to become single. In multiplayer games like the public goods one studied here, the situation may be different. In some cases, all the members of a group become single, while in some other cases, only those leaving become single and the left ones are still united together. Corresponding to these two different cases, we consider two dissociation mechanisms.

2.4 Under the first mechanism, there is no cohesiveness among group members. For a group of players involved in the repeated games, if anyone chooses to leave, all the members of this group become single and enter the matching pool. This is similar to the two-player game case, and all players in the matching pool are single. They are randomly regrouped to continue the subsequent rounds of the repeated game.

2.5 Under the second mechanism, there exists cohesiveness among group members. For the same group of players, only those choosing to leave would be single but the remaining ones would be united together. For convenience, we call them a union. So in the matching pool, single players and unions of various sizes may coexist. The matching process is a little more complex under this mechanism. In our main text, we assume that unions are first grouped with single players. Some general discussions are made in Appendix A. We firstly fill the unions with single players and then randomly regroup the rest of single players. For a union of size $k, k \in\{2, \cdots, N-1\}, G-k$ single players are randomly chosen from the matching pool. So they form a group and leave the matching pool. Then we continue to another union until only single players exist in the matching pool. At last, all these single players are re-matched randomly to form groups to play games. ${ }^{1}$

2.6 The details of these two dissociation mechanisms are illustrated in Figure 1 .

2.7 We remark that, to simplify the analysis, there is no cost for the matching process. Players in the matching pool do not lose any wealth and the matching process is fast enough so that players do not pay any waiting cost to reenter the game.

2.8 Players' strategies include two parts: the cooperation part indicates whether to cooperate or defect in the current round of games; and the leaving part indicates her reactions toward her group members' defection. In our model, for each individual, the cooperation part is fixed. That is, each player either always cooperates or always defects; she never changes actions in her life. The leaving part is a trigger strategy. To be specific, player $i$ 's strategy is expressed as a tuple $\left(p_{i}, q_{i}\right) \in \Sigma=\{C, D\} \times\{0,1, \ldots, G-1\}$ (so there are $2 G$ different strategies in total), where $p_{i}=C$ if the player cooperates and $p_{i}=D$ otherwise, and $q_{i}$ is the number of defective opponents a player can tolerate, i.e., if there are more than $q_{i}$ defectors among her opponents, then player $i$ leaves. Players are simple-minded in that they do not change their strategies during their lifetimes. Their strategies do not depend on any tags or reputations and the memory size is one. So the requirement for players' cognitive abilities is rather low.

2.9 The selection dynamic is similar to the Moran process. After each round of interaction, every individual dies with probability $\xi$. Hence, the lifespan of an individual is geometrically distributed with mean value $\alpha=\frac{1}{\xi}$. The death of an individual makes her group dismiss and the group members enter the matching pool. Without 

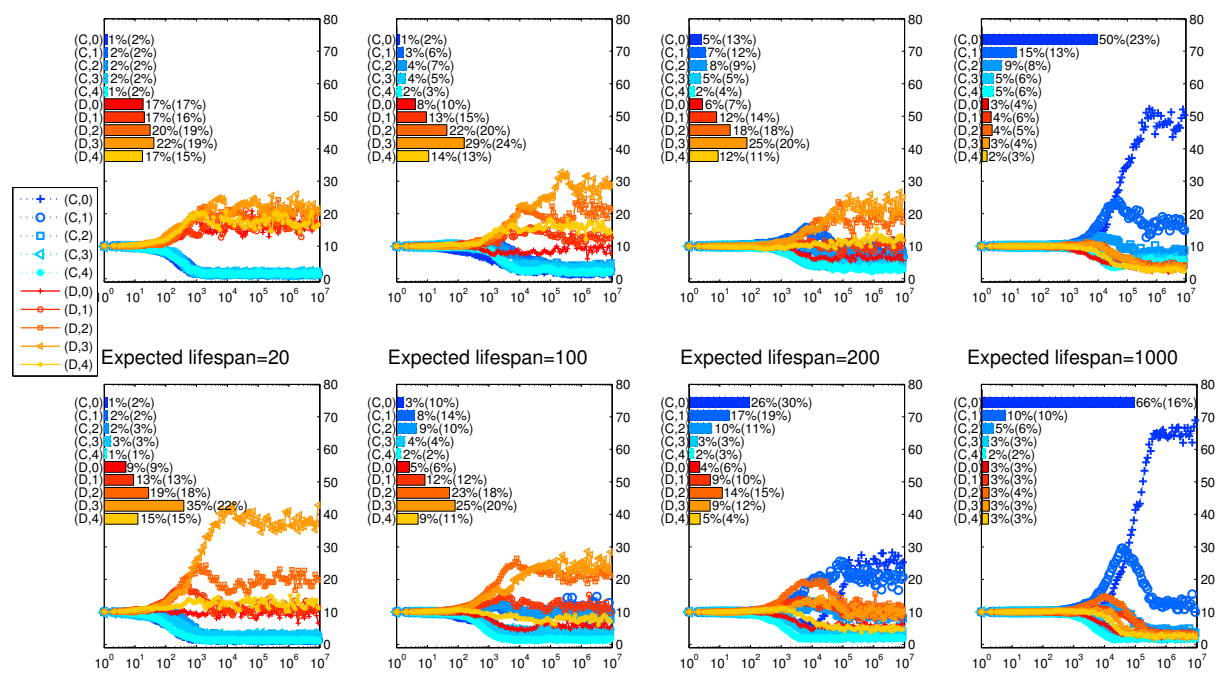

Figure 2: The evolution of strategies in $10^{7}$ rounds. The horizontal bar chart in the northwest of each panel is the strategy distribution at the final round and the data in bracket is the standard deviation. The top four panels are the outcomes of mechanism I that there is no cohesion and the bottom four are for mechanism II that cohesion exists. From left to right, the expected lifespans are 20,100, 200 and 1000, respectively. Each value is the average of 100 simulations. Parameters are: multiplication factor $r=3$, group size $G=5$, population size $N=200$, mutation rate $\mu=0.05$, selection strength $s=1$, which corresponds to the standard Moran process.

cohesiveness, under the first mechanism, if one player dies, all her surviving group-mates would become single. With cohesiveness, under the second mechanism, if one player dies, all her surviving group-mates who do not leave would form a union. Each individual who dies is replaced by a new entrant, so the population size remains unchanged. The new entrants would be single players and put into the matching pool. So in the matching pool, the total number of players is always divisible by $G$. The strategy of the new entrant is determined either by mutation or by imitation. If mutation happens, which occurs with a fixed probability of $\mu$, the new entrant would randomly choose one of the $2 G$ strategies. Otherwise, she imitates one of the surviving individuals' strategies, and the probability that one individual's strategy is imitated is proportional to her fitness, which is defined as

$$
f_{i}=w_{i}^{s}
$$

where $w_{i}$ is the wealth player $i$ has gained since she was born and $s$ is the selection strength of the dynamics. When $s=0,1$ and $\infty$, it equals the neutral drift, standard Moran process and best response dynamics respectively.

2.10 The above evolution dynamics defines a finite, irreducible, and aperiodic Markov chain. So a unique stationary distribution exists. ${ }^{2}$

\section{Simulation Results}

3.1 We run two series of simulations to investigate how the two different dissociation mechanisms affect the evolution of cooperation in the public goods game. ${ }^{3}$ From Izquierdo et al. (2014 2010), we know the expected lifespan of individuals determines whether conditional dissociation promotes cooperation. In this paper, we compare the two mechanisms for different lifespans too. In Figure 2, we present how the strategy distributions evolve and the main findings are summarized as follows.

i Longer lifespans promote cooperation.

It can be seen from Figure2 2 that, both dissociation mechanisms promote cooperation as the expected lifespan increases. When the expected lifespan of the agents is 20 , defectors dominate the population and cooperators can hardly survive under these two mechanisms. When the expected lifespan is 1000, cooperators dominate the population for both mechanisms. In the study of repeated games, while the folk's theorem 
tells us that cooperation may arise if the discount factor (the surviving rate here) is high enough, it doesn't explain how it arises.The above results show that conditional dissociation is an effective way to further promote the emergence of cooperation in repeated games.

ii Group cohesion results in higher cooperation level.

Comparing the top four panels with the bottom ones in Figure2, we find that cooperation is more abundant when cohesion exists. The most significant difference is observed when the expected lifespan is 200: with all other parameters fixed, more than half of the population are cooperators when cohesion exists while only about 30 percent are cooperators when there is no cohesion. This is due to the higher level of positive assortment among intolerant cooperators under mechanism II. As intolerant cooperators almost always appear in the matching pool, the more defectors in unions, the more likely that those intolerant cooperators be grouped with each other.

iii When cooperators dominate the population, strategy $(C, 0)$ is most abundant.

Just as shown in |zquierdo et al. (2010) and Qu et al. (2016), it is not surprising that the most intolerant strategies take the lead in the simulation when the players' expected lifespan is long enough. It is interesting that the flourish of strategy $(C, 0)$ always comes after the more tolerant strategy $(C, 1)$.

As there are initially equal numbers of defectors and cooperators and the death rate of players is low, there are about half defectors after 1000 rounds of games. These defectors are mainly in the matching pool, making it hard to find a group of cooperators. Apparently, for the same strategy distributions, the more tolerant strategies can form stable groups earlier at the cost of being exploited. Compared with returning to the matching pool and meeting other players, to tolerate a defector pays off. As the number of $(C, 1)$ strategists increases, the $(C, 0)$ strategists gain advantage and excess the $(C, 1)$ strategists.

iv When defectors dominate the population, the most successful strategy is $(D, 3)$.

While cooperators can hardly survive under these scenarios, mutations change some individuals into cooperators occasionally. So cooperators are mainly mutants and first appear in the matching pool. For a player to gain more, she should spend more time to play with cooperators. In practice, a successful defector needs to do two things. Firstly, she should leave her group immediately if all of her group members are defectors. If a defector is too tolerant e.g. $(D, 4)$, she has a smaller chance to enter the matching pool, and thus less likely to meet with cooperators. Secondly, once having encountered even one cooperator, a player should not leave because cooperators are rare, it is almost impossible to find a group containing two or more cooperators. From this point of view, $(D, 0),(D, 1)$, and $(D, 2)$ strategies are too intolerant. On the other hand, unless the mutant strategy is $(C, 4)$, almost no player can play with the same cooperator in the next round. When it happens, a group of one $(C, 4)$ and three $(D, 3)$ or $(D, 4)$ players can last for some rounds. So the $(D, 3)$ strategy gains its advantage over $(D, 0),(D, 1)$, and $(D, 2)$ when they meet with $(C, 4)$ players. This conjecture is justified in Figure 5 , where strategy $(C, 4)$ is made unavailable for players.

3.2 In the sequel we will provide some theoretical analyses to ensure the robustness and more importantly to better understand the dissociation mechanisms. In these analyses, we usually consider only two strategies: one is strategy $(C, 0)$ and the other is a defective strategy. This would simplify the matching problem in the pool significantly as the strategy distribution would be a Binomial distribution. However, for the last two findings, a theoretical analysis becomes extremely harder. This is because when we do that, at least three or more strategies should be considered, which lead to multiple equations. In addition to that, with more strategies also means the strategy distributions in the matching pool become a Multinomial distribution which also makes the problem even more difficult.

\section{Analytical Results}

4.1 Although some simplifications have been operated, it is still hard to make a complete analysis of the evolution dynamics due to the complexity of the leaving and rematching processes. In this section, we are going to prove the following three statements:

1. Only defective strategies can be NSS;

2. Longer expected lifespan favors cooperation;

3. Group cohesion promotes cooperation. 


\section{Only defective strategies can be NSS}

4.2 It is easy to see that no strategy is an evolutionarily stable strategy as each cooperative (or defective) strategy is neutrally against another cooperative (or defective) strategy. So we just need to discuss whether neutrally stable strategies (NSS) exist. According to Weibull (1997)'s definition, a strategy is an NSS if when the frequency of the invading strategies is small enough, the invaders could not earn greater payoffs than that of the incumbent strategy.

4.3 Before we continue on our analysis, we recall that unlike Fujiwara-Greve \& Okuno-Fujiwara 2009) and Rob \& Yang (2010) whose analyses are conducted on the full strategy spaces of the repeated prisoner's dilemma game, the strategy space considered here is rather simple and limited. In our paper, the strategy space is defined as $\Sigma=\{C, D\} \times\{0,1, \ldots, G-1\}$, which contains no mixed strategies. We also assume continuous populations in the following to simplify our analysis. We first prove that all defective strategies are NSSs and then that no cooperative strategy is NSS.

4.4 Firstly, when the incumbent strategy is a defective strategy and the proportion of invading cooperative strategy is small enough, the expected payoffs of invading cooperators would be about $\frac{r}{G \xi}$ as they are almost always playing games with defectors, while the expected payoffs of defectors are $\frac{1}{\xi}$. Since $\frac{r}{G \xi}<\frac{1}{\xi}$, and each defective strategy is neutral to each other, we see that the focal defective strategy is an NSS.

4.5 Secondly, when the incumbent strategy is a cooperative strategy and the proportion of invading defective strategy is small enough, then the payoffs to the invading defectors would be about $\frac{r(G-1)}{G \xi}+\frac{1}{\xi}$, which is larger than $\frac{r}{\xi}$, the payoffs of cooperators. So the invading defectors get more than the incumbent cooperators. That is, no cooperative strategy could be an NSS.

\section{Longer expected lifespan favors cooperation}

4.6 In this part, for simplicity, we consider the case that no cohesion exists and assume that the cooperative strategy is $(C, 0)$ and the defective strategy is any one but $(D, 4)$.

4.7 Since no cohesion exists, and defectors could not endure a group with no cooperators, at the beginning of each round, all the defectors appear in the matching pool as single players. For a cooperator, she may either appear in the matching pool as a single player or stay in her group with other $G-1$ cooperators. Let $x_{g}$ denote the proportion of cooperators who are in groups, $x_{s}$ those in the pool and $y$ the proportion of defectors $\left(x_{s}+x_{g}+\right.$ $y=1$ ). Then the mean-field equations for $x_{g}, x_{s}$ and $y$ in the large populations are:

$$
\begin{aligned}
\dot{x_{g}} & =\left(x_{g}+\frac{x_{s}^{G}}{\left(x_{s}+y\right)^{G-1}}\right)(1-\xi)^{G}-x_{g} \\
\dot{x_{s}} & =\left(x_{g}+\frac{x_{s}^{G}}{\left(x_{s}+y\right)^{G-1}}\right)\left(1-\xi-(1-\xi)^{G}\right)+x_{s}\left(1-\left(\frac{x_{s}}{x_{s}+y}\right)^{G-1}\right)(1-\xi)+\frac{\xi \cdot \pi_{c}}{\pi_{c}+\pi_{d}}-x_{s} \\
\dot{y} & =y(1-\xi)+\frac{\xi \cdot \pi_{d}}{\pi_{c}+\pi_{d}}-y
\end{aligned}
$$

where $\pi_{c}$ and $\pi_{d}$ are the total payoffs of cooperators and defectors respectively. A more detailed explanation of the above equations are given in Appendix F here we only briefly introduce how to get $\dot{x_{g}}$. In each round of games, some single cooperators might form new cooperative groups. And the proportion of players in the newly formed cooperative groups is $\frac{x_{s}^{G}}{\left(x_{s}+y\right)^{G-1}}$. So the total proportion of cooperators in cooperative groups is $x_{g}+\frac{x_{s}^{G}}{\left(x_{s}+y\right)^{G-1}}$. For a cooperative group, the probability that none of the group members dies in the current round is $(1-\xi)^{G}$. Thus in the end of the current round (also the beginning of the next round), the proportion of cooperators in groups is $\left(x_{g}+\frac{x_{s}^{G}}{\left(x_{s}+y\right)^{G-1}}\right)(1-\xi)^{G}$ and then we get $\dot{x_{g}}$.

4.8 To fully describe the dynamics, we now calculate $\pi_{c}$ and $\pi_{d}$. In our model, players' payoffs are accumulated since they were born. As the strategy distribution changes over time, it is hard to calculate players' payoffs from the beginning of the evolution. However, when the population evolve near to the stationary strategy distribution, we can calculate their payoffs approximately. For a cooperator if she is in a cooperative group, her payoffs are $r$; if she is single she would be regrouped with another $G-1$ single players whose strategy distribution is a Binomial distribution $B\left(G-1, \frac{x_{s}}{x_{s}+y}\right)$ and her expected payoff would be $\frac{1}{G} \cdot\left(1+\frac{(G-1) x_{s}}{x_{s}+y}\right) r$. 


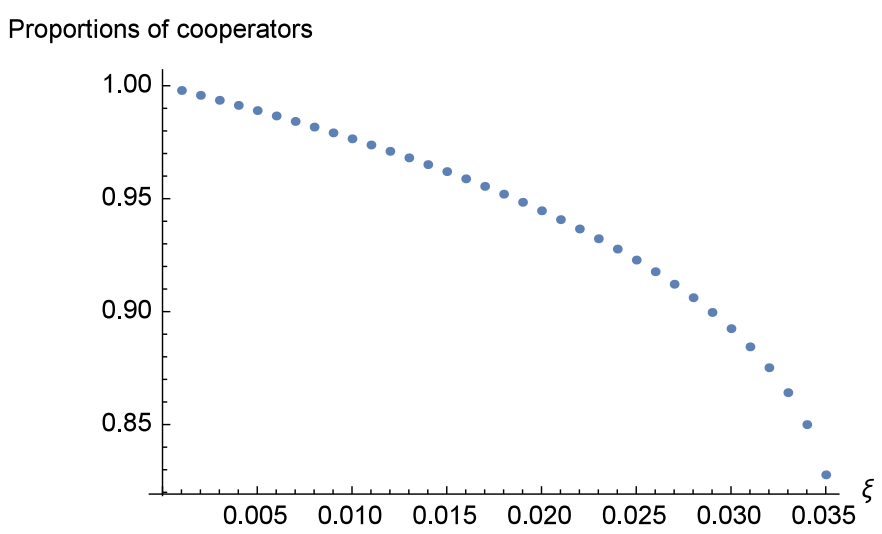

Figure 3: The proportions of cooperators at the stable rest states decrease for increasing $\xi$ s. Parameters are $G=5$ and $r=3$.

So at a stable interior rest point (if exists), the average expected total payoffs for cooperators and defectors are, respectively,

$$
\begin{aligned}
& \pi_{c}=\frac{1}{\xi} \cdot\left(\frac{\left(G x_{s}+y\right) x_{s} r}{G\left(x_{s}+y\right)}+x_{g} r\right), \\
& \pi_{d}=\frac{1}{\xi} \cdot\left(\frac{(G-1) x_{s} r y}{G\left(x_{s}+y\right)}+y\right) .
\end{aligned}
$$

4.9 While it is impossible to get full solutions of the above system defined by Equations 1 and 2 (which are equivalent to a series of fifth and higher degree equations) in general, numerical methods suggest that the system has a stable interior rest point when $\xi \leq \xi^{*}$ (for our parameters, $\xi^{*}$ is about 0.035 ), and as $\xi$ increases and no more than $\xi^{*}$, the proportion of cooperators shrinks as can be seen in Figure 3 . Because the expected lifespan of players is the reciprocal of $\xi$, cooperation is enhanced when expected lifespan increases.

\section{Group cohesion favors cooperation}

4.10 In this section, we prove that the expected number of cooperators that a cooperator may meet is larger when cohesiveness exists.

4.11 Under mechanism I, all the players in the matching pool are single. Because the matching process is randomly conducted, the group members' distribution is a binomial distribution. The probability for a cooperator to meet with $k(k \leq G-1)$ cooperators is

$$
P_{k}=\left(\begin{array}{c}
G-1 \\
k
\end{array}\right) \frac{x_{s}^{k} y^{(G-1-k)}}{\left(x_{s}+y\right)^{(G-1)}} .
$$

And the expected number of cooperators she can meet is $E_{1}^{c}=\frac{(G-1) x_{s}}{x_{s}+y}$.

4.12 Under mechanism II, there are three kinds of players in the matching pool: single cooperators, single defectors and unions of defectors. Let $y_{k}$ denote the proportions of players in $k$-unions $(2 \leq k \leq G-1)$ and $y_{s}$ the proportion of single defectors, so we have $y=y_{s}+\sum_{k=2}^{G-1} y_{k}$.

4.13 For each $k$-union, $G-k$ single players are needed and their strategy distribution is a Binomial distribution $B\left(G-k, \frac{x_{s}}{x_{s}+y_{s}}\right)$. After the unions have all been regrouped, the single players are going to be regrouped, and the strategy distribution of each group is also a Binomial distributions $B\left(G, \frac{x_{s}}{x_{s}+y_{s}}\right)$. Denote $p_{k}$ the probability that a single player would be grouped with a $k$-union. Obviously, it depends on the number of $k$-unions and

$$
p_{k}=\frac{y_{k}(G-k)}{k\left(x_{s}+y_{s}\right)}
$$


The expected number of cooperators that a cooperator may meet is

$$
\begin{aligned}
E_{2}^{c} & =\sum_{k=2}^{G-1} p_{k} \cdot(G-k-1) \cdot \frac{x_{s}}{x_{s}+y_{s}}+\left(1-\sum_{k=2}^{G-1} p_{k}\right) \cdot(G-1) \cdot \frac{x_{s}}{x_{s}+y_{s}} \\
& =\frac{x_{s}}{x_{s}+y_{s}}\left[(G-1)-\sum_{k=2}^{G-1} p_{k} k\right] \\
& =\frac{x_{s}}{x_{s}+y_{s}}\left[(G-1)-\sum_{k=2}^{G-1} \frac{y_{k}(G-k)}{x_{s}+y_{s}}\right]
\end{aligned}
$$

4.14 For fixed $x_{s}$ and $y$, to prove $E_{1}^{c}<E_{2}^{c}$, we just need to prove $E_{2}^{c}-E_{1}^{c}>0$.

$$
\begin{aligned}
E_{2}^{c}-E_{1}^{c} & =\frac{x_{s}(G-1)\left(y-y_{s}\right)}{\left(x_{s}+y_{s}\right)\left(x_{s}+y\right)}-\frac{x_{s}}{\left(x_{s}+y_{s}\right)^{2}} \sum_{k=2}^{G-1} y_{k}(G-k) \\
& =\frac{x_{s}(G-1) \sum_{k=2}^{G-1} y_{k}}{\left(x_{s}+y_{s}\right)\left(x_{s}+y\right)}-\frac{x_{s} \sum_{k=2}^{G-1} y_{k}(G-k)}{\left(x_{s}+y_{s}\right)^{2}} \\
& =\frac{x_{s}}{x_{s}+y_{s}}\left[\frac{(G-1) \sum_{k=2}^{G-1} y_{k}}{x_{s}+y}-\frac{\sum_{k=2}^{G-1} y_{k}(G-k)}{x_{s}+y_{s}}\right] \\
& =\frac{x_{s}}{x_{s}+y_{s}} \sum_{k=2}^{G-1} y_{k}\left(\frac{G-1}{x_{s}+y}-\frac{G-k}{x_{s}+y_{s}}\right)
\end{aligned}
$$

Let

$$
\triangle=\sum_{k=2}^{G-1} y_{k}\left(\frac{G-1}{x_{s}+y}-\frac{G-k}{x_{s}+y-\sum_{k=2}^{G-1} y_{k}}\right)
$$

Then

$$
\begin{aligned}
\frac{\partial \triangle}{\partial y_{k}} & =\frac{G-1}{x_{s}+y}-\frac{G-k}{x_{s}+y-\sum_{k=2}^{G-1} y_{k}}-\frac{(G-k) \sum_{k=2}^{G-1} y_{k}}{\left(x_{s}+y-\sum_{k=2}^{G-1} y_{k}\right)^{2}} \\
& =\frac{G-1}{x_{s}+y}-\frac{(G-k)\left(x_{s}+y\right)}{\left(x_{s}+y-\sum_{k=2}^{G-1} y_{k}\right)^{2}}
\end{aligned}
$$

It is easy to verify that

$$
\left.\frac{\partial \triangle}{\partial y_{k}}\right|_{\sum_{k=2}^{G-1} y_{k}=0}=\frac{G-1}{x_{s}+y}-\frac{G-k}{x_{s}+y}=\frac{k-1}{x_{s}+y}>0
$$

Since

$$
E_{2}^{c}-E_{1}^{c} \mid \begin{gathered}
\sum_{k=2}^{G-1} y_{k}=0 \\
=0
\end{gathered}
$$

we know for sufficiently small $y_{k}$,

$$
E_{2}^{c}-E_{1}^{c}>0
$$

While it is hard to give the exact proportion of players in unions during the repeated games, we can learn from the above analysis that cooperators are more likely to meet each other when some defectors are united together. Actually, from Equation 3 , we see that when

$$
\sum_{k=2}^{G-1} y_{k}<\frac{x_{s}+y}{G-1}, E_{2}^{c}-E_{1}^{c}>0 .
$$

4.15 Based on the above simulation and theoretical results, we can explain the differences of the two mechanisms in promoting cooperation. Since strategy $(C, 0)$ is the only cooperative strategy that may win the evolution, the differences of the two mechanisms are essentially how they promote the evolution of strategy $(C, 0)$. Under both mechanisms, the players using $(C, 0)$ would leave their current groups whenever they meet defectors. So in the matching pool, all players using $(C, 0)$ are single players. For a player using $(C, 0)$, she could meet other 
$(C, 0)$ strategists only when she is grouped with single players. Under the first mechanism, all players in the matching pool are single players. But under the second mechanism, some players are in unions. For the same strategy distribution, there would be more single players in the matching pool under the first mechanism than under the second mechanism. However, there should be equal amount of $(C, 0)$ strategists in the matching pool. As a result, the single $(C, 0)$ players are more likely to be regrouped with each other under the second mechanism. That is, cohesion brings about a stronger positive assortment effect, and thus promotes a higher level of cooperation.

\section{Discussion}

5.1 We have studied how cooperation evolves in public goods games under conditional dissociation mechanisms and investigated the role of group cohesion. We have compared two dissociation mechanisms, one with cohesion and the other without. Our results show that both mechanisms promote the evolution of cooperation if the expected lifespan of the players is long enough. The dissociation mechanisms promote cooperation from two aspects. Firstly, the intolerant cooperators have a greater chance to find new cooperative partners. Secondly, once they meet a group of cooperators, the expected periods of the repeated games they play together are longer too. Both effects increase the probability that cooperators play games together. From this point of view, conditional dissociation can also be classified as a kind of positive assortment. Moreover, we find that the existence of cohesion can lead to significant higher levels of cooperation. This is because positive assortment effect is stronger when cohesion exists.

5.2 In game theory studies, cohesion is seldom studied. Unlike Jaffe (2006) and Jaffe \& Zaballa (2010) just treating the social cohesion as levels of cooperation, we regard cohesion as the tendency that a group of players would stay together after someone's occasional leaving. In psychological studies (Petersen et al.|2004), cohesion is usually measured by asking the subjects a series of questionnaires concerning their feelings and attitudes towards their groups. Then they compare how subjects behave in groups with different cohesion scores. While this method gives us a vivid figure of the correspondence between cohesion and people's performance, they can hardly tell us how it functions. Despite of capturing the point of staying together as mentioned in Festinger (1950)'s definition, our definition does not capture all successive modifications developed by other psychologists. Hogg (1993) and Hogg \& Hains (1996) pay more attention to the psychological essence of cohesion and claim cohesion is rooted in self-categorization. Bettencourt et al. (1997) find that increased cohesion is closely associated with ingroup favoritism. The existence of ingroup favoritism and its effect on cooperation is controversial and attracted lots of studies. Balliet et al. (2014) conduct a meta-analysis and confirm the existence of ingroup favoritism effect. They find evidence that categorization would result in ingroup favoritism. However, they also find that this effect is even stronger under structured situations, which supports reciprocity theory. Combining these studies together, we see another clue of how cohesion interacts with group performances. That is, as a result of self-categorization, cohesion leads to more intragroup interactions and the reciprocity mechanism works to promote cooperation and in turn ingroup favoritism.

5.3 It is noteworthy that the increase of players' expected lifespan also means the deathrate of players is lower. So for a group of players, the probability that they continue playing games together is higher, which implies higher cohesiveness too. In previous study of repeated games, the discounting factor is interpreted as players' patience Fudenberg \& Maskin 1986). Our work shows that it can also be interpreted as the cohesiveness of groups. To elaborate this point, we conduct another series of simulations where a group may disband by mistake. The mistake could be viewed as a result of poor cohesiveness. And we find (see Figure 6 ) that as the probability of mistakenly breaking up increases, cooperation is less promoted. Of course, the connotation of cohesion is rich. Our paper only considers one aspects of it. Studies investigating other aspects of cohesion would be good directions in future.

5.4 While our paper does not consider the full strategy space as Fujiwara-Greve \& Okuno-Fujiwara (2009) and Rob \& Yang (2010) do, it is natural to ask whether some of our results are robust to the trust-building strategies found in these studies. As is known, trust-building strategies cannot succeed when the expected lifespan is too short. So with short enough lifespans, cooperation cannot emerge even trust-building strategies are considered. On the other hand, when the expected lifespan is long enough, then both trust-building strategies and $(C, 0)$ strategy users get similar payoffs in the long run, which means they are neutral to each other. When the expected lifespan is medium, the situation is complex. On one hand, the trust-building strategy users suffer less than $(C, 0)$ strategy users when confronted with defectors. On the other hand, they are also wasting there periods to play with defectors as they would not leave a defective group immediately as $(C, 0)$ strategists do. Combining these effects together, it is hard to figure out how the population evolve when more complex strategies are available. 
Moreover, as trust-building strategies all require longer memory sizes, the strategy space is extraordinarily large and the methods used in this paper are inefficient in handling the new problems.

5.5 Wubs et al. (2016) study Prisoner's Dilemma game too and refer to conditional dissociation as partner switching. They compare it with two other partner control mechanisms, the direct reciprocity and punishment mechanism. They find that when the population size is small, partner switching is less efficient than the other two mechanisms in promoting cooperation. However, as the population size increases, partner switching mechanism results in more cooperation than other mechanisms. In Appendix E(see Figure9), we obtain similar findings that a larger population size generates a higher proportion of cooperation.

5.6 In our model, it costs players nothing to leave their opponents and to be regrouped. Their private wealth would not decrease and they waste no time to engage in another round of interaction of the repeated games. If players pay some cost during the leaving process, cooperation is better promoted in Prisoner's Dilemma game when the cost is small (Izquierdo et al.|2010: Qu et al. 2016). An intuitive explanation is that defectors are more disliked, so they lose more. In public goods games, the situation may be more complex and beyond the scope of this paper. The followings are some of our considerations. Players would suffer more losses once they enter the matching pool as the probability to be grouped with satisfied members is much smaller in public goods games. Since the winning cooperative strategy is also the intolerant strategy, it suffers more. So cooperation may be suppressed. Moreover it is also problematic that whether players in unions should suffer the same losses as single players. If not, how the sizes of unions affect the losses is open to question too. So it requires more careful and comprehensive discussion and would be an interesting topic for further research.

5.7 Furthermore, previous works, e.g. Van Segbroeck et al. (2012) and Han et al. (2017); Martinez-Vaquero et al. 2015 have considered trigger strategies with a threshold in the context of repeated games. Namely, players can decide whether to cooperate in a next round of the game or stay in a long-term agreement depending on whether the number of cooperative co-players in the current round reaches a certain threshold. These works however do not allow the possibility that players can leave the group. On the other hand, the present work does not include such reciprocal strategies. It would be interesting to study how reciprocity and the leaving mechanisms interact and compete in the context of repeated games. For instance, whether it is necessary to leave a group in which there are many defectors or it is better to act reciprocally instead, especially when leaving and rejoining another group are very costly.

\section{Notes}

${ }^{1}$ When unions exist in the matching pool, there are also other matching procedures to regroup the agents. In Appendix A we investigate another re-matching process where unions would be regrouped with unions and we obtain similar conclusions (see Figure 4).

${ }^{2}$ This does not mean the dynamics has a unique stable state corresponding to this stationary distribution.

${ }^{3}$ In our main text, we set $s=1, G=5, r=3, N=200$ for all the simulations, and outcomes for other parameters are presented in Appendix E (see Figures 912. And for different initial strategy distributions, the results are presented in Appendix D (see Figure 7 and 8 .

\section{Acknowledgments}

Our research is supported by the National Natural Science Foundation of China (NO. 71701058, NO. 71871009, NO. 71532013, NO. 71850008) and Future of Life Institute (grant RFP2-154).

\section{Appendix A: Different re-matching processes}

In the main text, the re-matching process of mechanism II does not allow unions to be regrouped with other unions. Yet, in reality, the contrary may occur too. In this part, we consider another re-matching process where unions are regrouped with unions firstly.

Let $k$-union denote a union that consists of $k$ players and $S_{k}$ denote the set of all the $k$-unions remained in the matching pool (Here we treat each single player as a 1-union). Let $L(k)=\max \left\{l \mid l \leq k, S_{l} \neq \varnothing\right\}$ be 
the size of the largest unions that remain in the matching pool with sizes no bigger than $k$. Let $M_{k}=S_{L(k)}$. The largest union in the matching pool is regrouped first. If there are more than one largest unions, then we randomly choose one of them with equal probabilities. Let $K$ be the size of the largest unions remaining in the matching pool. For the chosen $K$-union, it would be united with a randomly chosen union from $M_{G-k}$. For this newly formed union, if its size is $G$, then it forms a group and all its members leave the matching pool. Else if its size is smaller than $G$, it remains in the matching pool and the above matching process is continued. After a finite number of rounds of matchings, all the players in the matching pool will be regrouped. Then all the groups, either previously formed and or newly formed, play the public goods game simultaneously.

From Figure 4 , we can see that the main conclusions we get in our main text still hold for this matching process. Compared with Figure 2, we see that cooperation is better promoted under this new matching process. With this matching process, single players are more likely to meet single players, implying that $(C, 0)$ strategists are more likely to meet other $(C, 0)$ players.
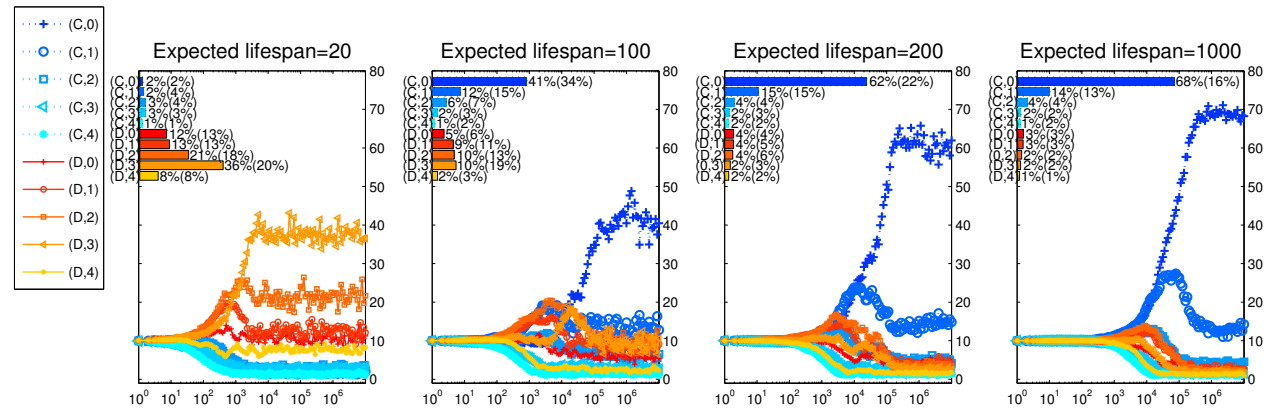

Figure 4: Strategy distribution after $10^{7}$ rounds of games with new re-matching process. Parameters are: group size $G=5$, multiplication factor $r=3$, population size $N=200$, mutation rate $\mu=0.05$, selection strength $s=1$.

\section{Appendix B: When strategy $(C, 4)$ is unavailable}

In these simulations, there are equal number of cooperators and defectors initially too. To achieve this setting, each of the other four cooperative strategies are endowed with 25 players. Moreover, when mutation happens, by a similar means, the probability to be a cooperator is also equal to be a defector. In the simulations, the expected lifespan of individuals are 20 and 100. The results are shown in Figure 5. It can be seen, the leading advantage of $(D, 3)$ is much smaller in these simulations, which can serve as a verification of our hypothesis. 

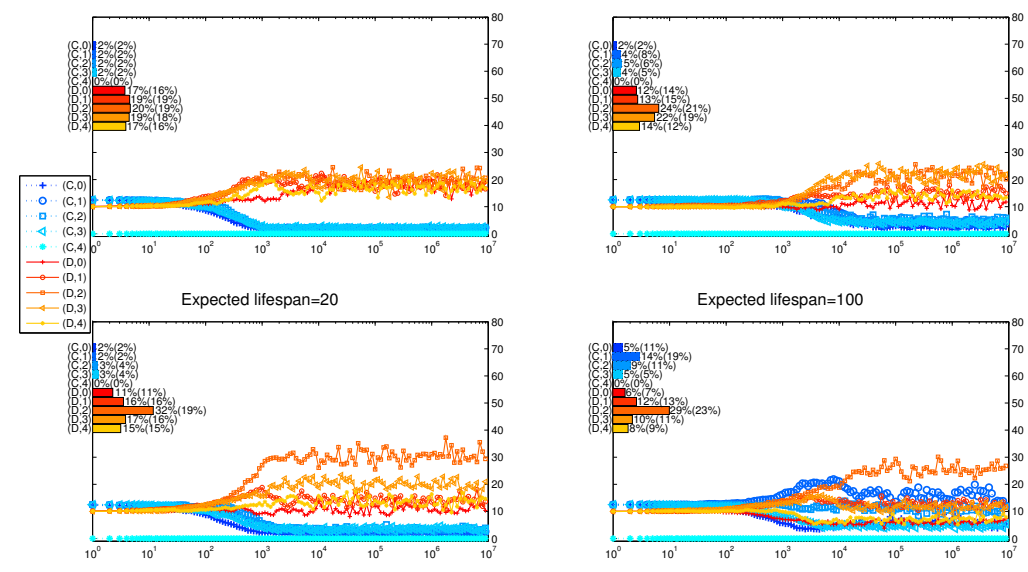

Figure 5: The details of the simulations without strategy $(1,4)$. The upper two graphs are the results for mechanism I and the bottom two are for mechanism II. From left to right, the expected lifespan are 20 and 100 respectively. Each graph is an average of 100 simulations. Parameters are: group size $G=5$, multiplication factor $r=3$, death rate $\xi=0.001$, mutation rate $\mu=0.05$, selection strength $s=1$, population size $\mathrm{N}=200$.

\section{Appendix C: Noise effect}

Figure 6 shows that the existence of noises causes severe detriments to cooperators. From the view of cohesion, noises can be seen as a lack of cohesion. The larger probability that noises happen, the less cohesion among the group members and cooperation.
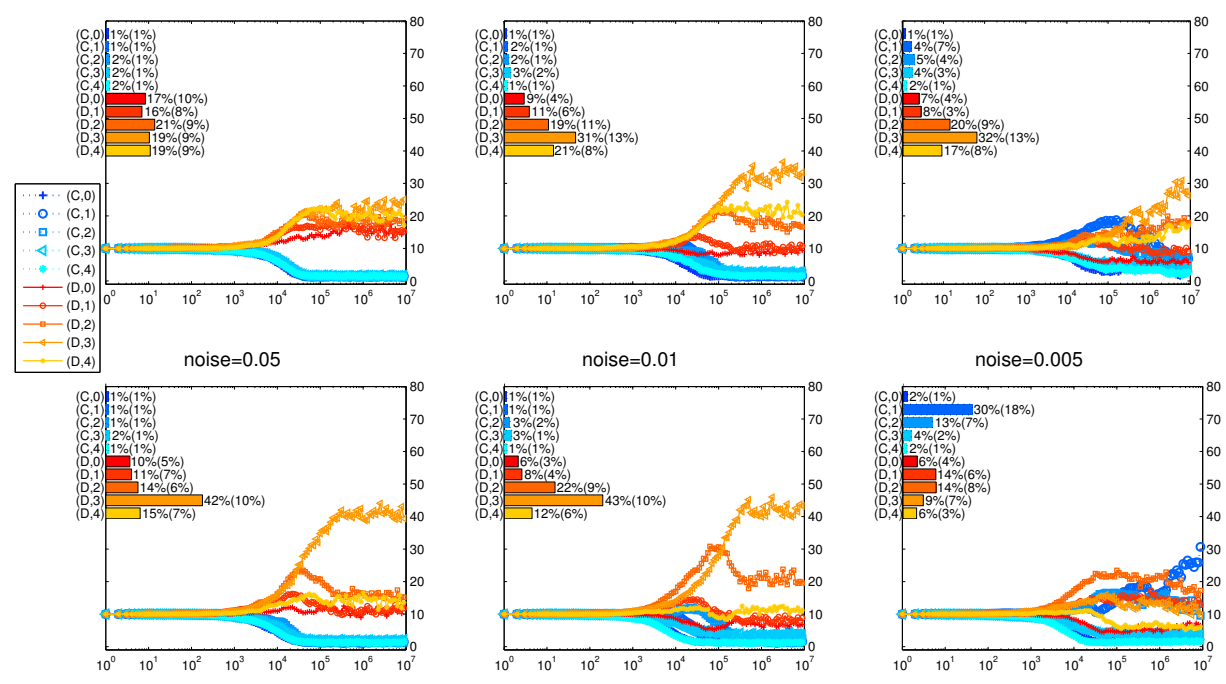

Figure 6: The details of the simulations when noises exist. The upper 3 panels are for mechanism I where no cohesion exists. And the bottom 3 are for mechanism II with cohesion. Noises happen with probability 0.05 , 0.01 and 0.005 respectively from the left to right. Other parameters are: group size $G=5$, multiplication factor $r=3$, death rate $\xi=0.001$, mutation rate $\mu=0.05$, selection strength $s=1$, population size $\mathrm{N}=200$.

\section{Appendix D: Different initial strategy distributions}

In Figure 7 and Figure 8 , we present the details of simulations with different initial distributions of strategies. In each figure, the upper 2 panels are for mechanism I and the bottom 2 panels are for mechanism II; the left 
2 panels are simulations initialized with $\frac{2}{5}$ agents being cooperators while the right 2 panels are simulations initialized with $\frac{3}{5}$ agents being cooperators. We can see from these two figures that despite the differences in initial proportions of cooperators, the simulations end up with similar strategy distributions after $10^{7}$ rounds of games given all other settings being the same.
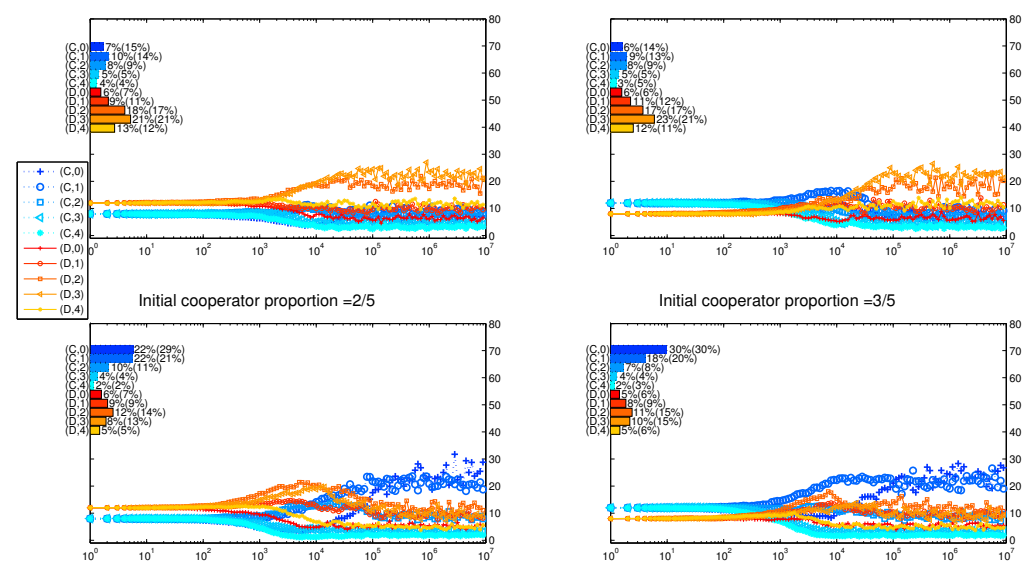

Figure 7: Expected lifespan is 200
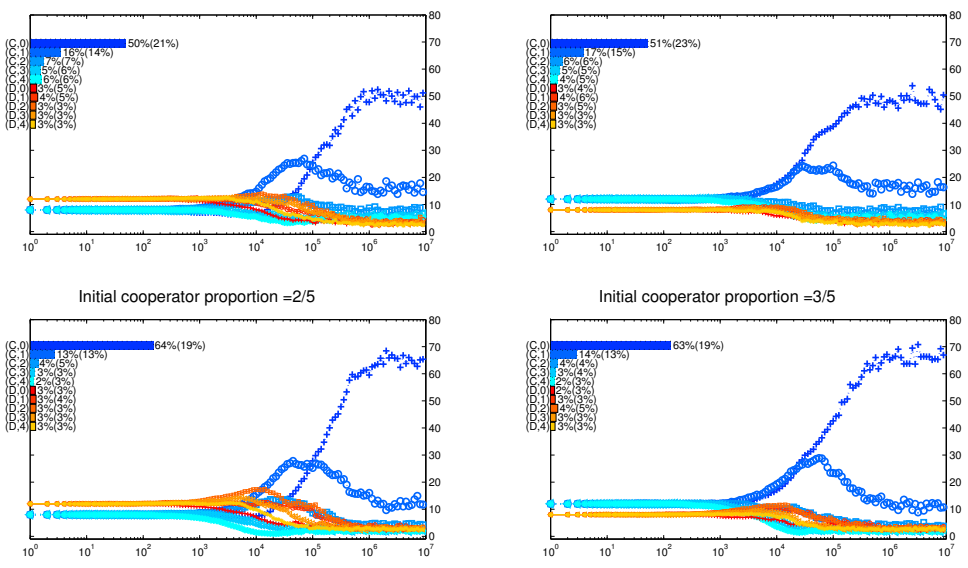

Figure 8: Expected lifespan is 1000

\section{Appendix E: Other different parameters}

From Figure 9, we can see that when population size increases, cooperation is promoted. This finding agrees with (Wubs et al.2016) that dissociation mechanism promotes cooperation better when population size is even larger. 

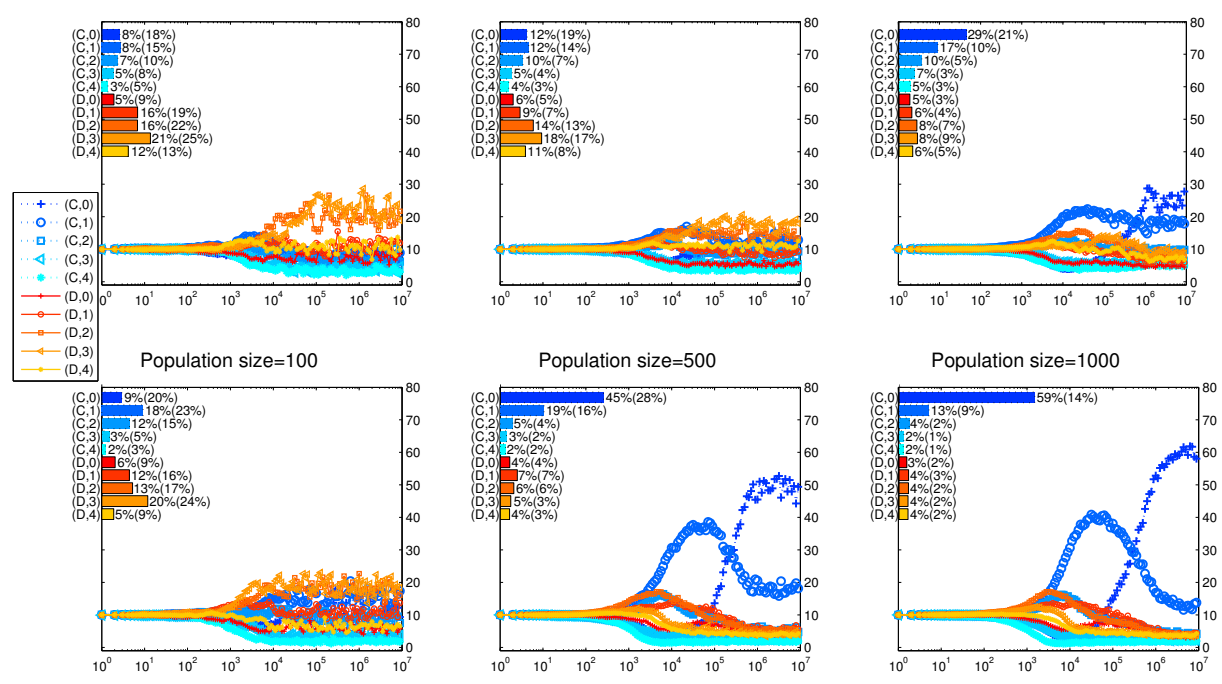

Figure 9: The details of the simulations with different population sizes. The upper 3 panels are for mechanism I where no cohesion exists. And the bottom 3 are for mechanism II with cohesion. The population sizes are 100, 500 , and 1000 respectively from the left to right. And other parameters are: group size $G=5$, multiplication factor $r=3$, death rate $\xi=0.005$, mutation rate $\mu=0.05$, selection strength $s=1$.
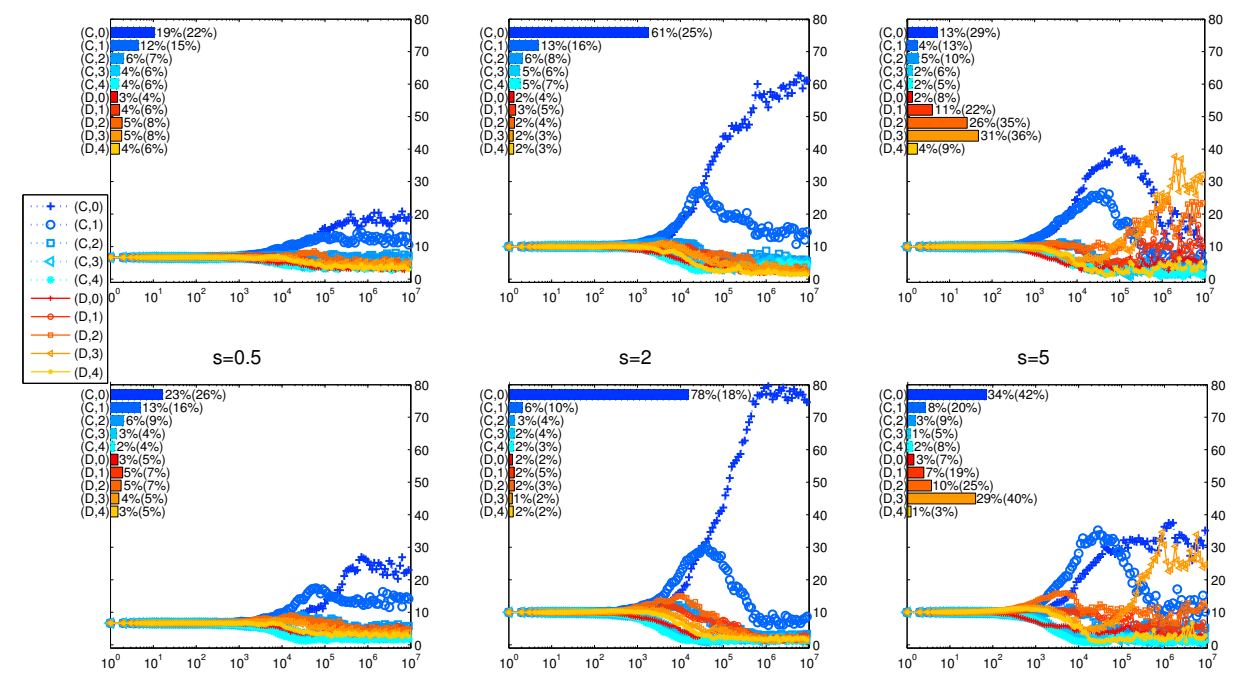

Figure 10: The details of the simulations with different selection strengths. The upper 3 panels are for mechanism I, and the bottom 3 are for mechanism II. Selection strengths from the left to the right are $s=0.5, s=$ $2, s=5$ respectively. Other parameters are: group size $G=5$, multiplication factor $r=3$, death rate $\xi=0.001$, mutation rate $\mu=0.05$, population size $\mathrm{N}=200$.

In Figure 10, we observe that, our results are robust when the selection strengths are not too strong (see, $s=0.5$ and $s=2$ ). For stronger selections (see $s=5$ ), defective strategies have a greater chance to survive. However, cooperative strategies still have larger frequencies under cohesion (Mechanism II) than when it is absent (Mechanism I). Moreover, it is noteworthy that, behavioral experiments with real human subjects in various contexts have confirmed that selection strengths are usually not excessively strong (see e.g. Rand et al. (2013); Zisis et al. (2015).

It has long been proved (Szabó \& Fath 2007) that in public goods game, cooperators gain more advantages with 
smaller group sizes or larger multiplication factor, and Figure 11 and 12 show that this conclusion still holds in our model.
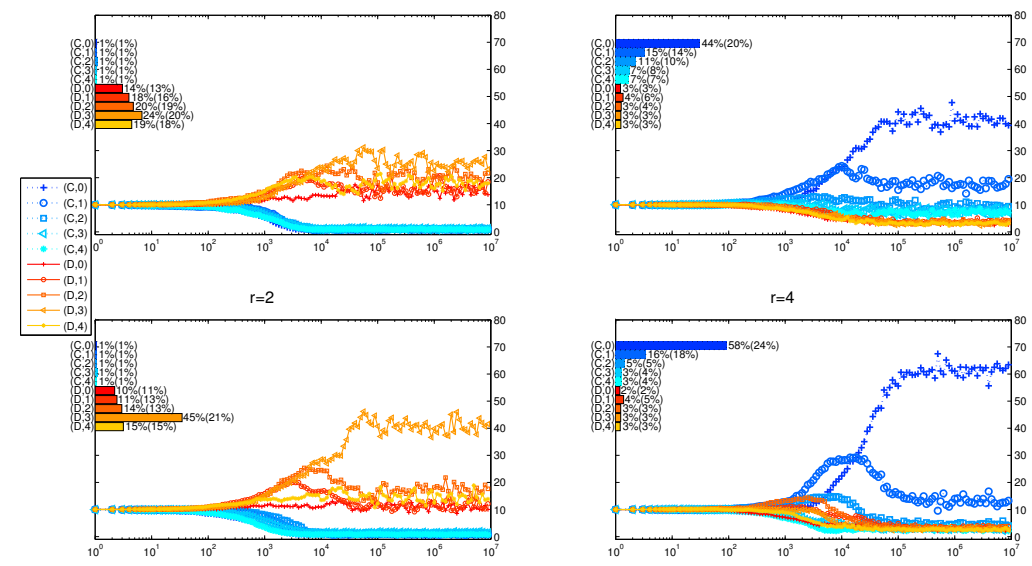

Figure 11: The details of strategy evolution with different value of $r$. The upper 2 panels are for mechanism I, and the bottom 2 are for mechanism II. The value of $r$ are 2 for the left two panels and 4 for the right 2. Other parameters are: group size $G=5$, death rate $\xi=0.005$, mutation rate $\mu=0.05$, selection strength $s=1$, population size $\mathrm{N}=200$.
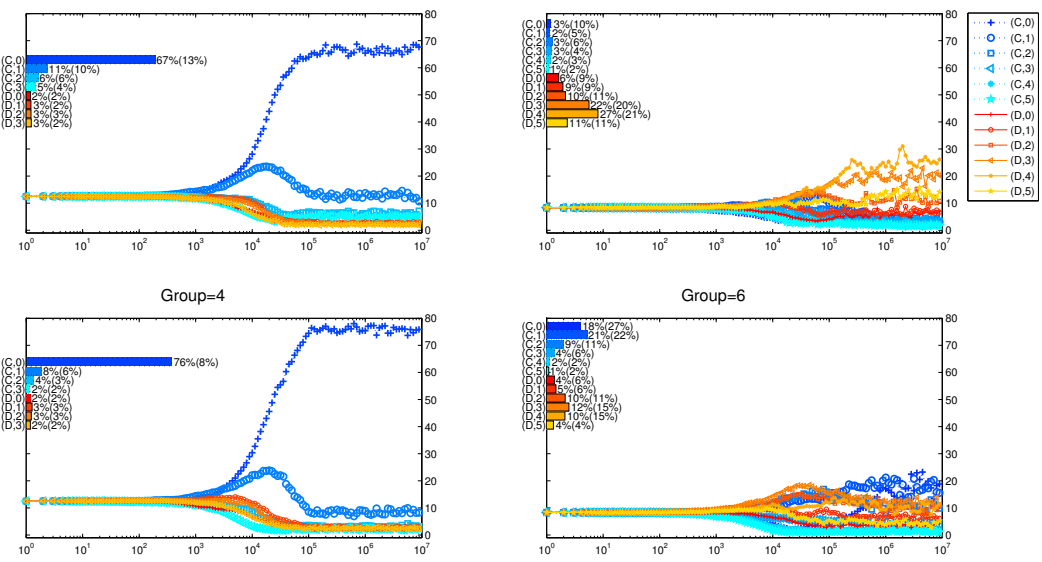

Figure 12: The details of strategy evolution in $10^{7}$ rounds of games with different group size. The upper 2 panels are for mechanism I, and the bottom 2 are for mechanism II. The sizes of groups are 4 for the left two panels and 6 for the right 2. Other parameters are: multiplication factor $r=3$, death rate $\xi=0.005$, mutation rate $\mu=0.05$, selection strength $s=1$, population size $\mathrm{N}=200$.

\section{Appendix F: Explanation of Equation 1}

Let $x_{g}(t), x_{s}(t), y(t)$ be the proportion of cooperators who are in a cooperative groups, single cooperators and single defectors in the matching pool at the beginning of round $t$. Then single players are randomly regrouped and play games. After some players' leaving and death, the proportions change.

Now let us calculate $x_{g}(t+1), x_{s}(t+1), y(t+1)$.

$x_{g}(t+1)$, players who are in cooperative groups at the beginning of round $t+1$, are constituted of two kinds of players. Some of them are in fully cooperative groups and some are single at the beginning of round $t$ but are then grouped together in that round, with which the proportion is $\frac{x_{s}^{G}(t)}{\left(x_{s}(t)+y(t)\right)^{G-1}}$. Since the probability that 
a single player dies in each round of games is $\xi$, for a group of cooperators, they would survive round $t$ with a probability of $(1-\xi)^{G}$. Putting these two sources together, we get

$$
x_{g}(t+1)=\left(x_{g}(t)+\frac{x_{s}^{G}(t)}{\left(x_{s}(t)+y(t)\right)^{G-1}}\right)(1-\xi)
$$

$x_{s}(t+1)$, single cooperators are constituted of three kinds of players. Firstly, some of them are those who have been in cooperative groups at round $t$ but unfortunately some of the group members die. Since the proportion of players who have been in cooperative groups is $x_{g}(t)+\frac{x_{s}^{G}(t)}{\left(x_{s}(t)+y(t)\right)^{G-1}}$, and some groups survive to round $t$ with probability $(1-\xi)^{G}$, the proportion of surviving single players is $\left(x_{g}(t)+\frac{x_{s}^{G}(t)}{\left(x_{s}(t)+y(t)\right)^{G-1}}\right)(1-\xi-(1-$ $\left.\xi)^{G}\right)$. Secondly, some of them are those who are single and meet some defectors and survive to round $t+1$. And the proportion of this kind of players is $x_{s}\left(1-\left(\frac{x_{s}(t)}{x_{s}(t)+y(t)}\right)^{G-1}\right)(1-\xi)$. Finally, some newborn players are being cooperators, and the proportion is $\frac{\xi \cdot \pi_{c}}{\pi_{c}+\pi_{d}}$. So

$x_{s}(t+1)=\left(x_{g}(t)+\frac{x_{s}^{G}(t)}{\left(x_{s}(t)+y(t)\right)^{G-1}}\right)\left(1-\xi-(1-\xi)^{G}\right)+x_{s}\left(1-\left(\frac{x_{s}(t)}{x_{s}(t)+y(t)}\right)^{G-1}\right)(1-\xi)+\frac{\xi \cdot \pi_{c}}{\pi_{c}+\pi_{d}}$

$y(t+1)$, single defectors are constituted of two kinds of players. Some are those surviving to round $t+1$ and some are newborn ones. The proportion of these two kinds of defectors are $y(t)(1-\xi)$ and $\frac{\xi \cdot \pi_{d}}{\pi_{c}+\pi_{d}}$ respectively. So

$$
y(t+1)=y(t)(1-\xi)+\frac{\xi \cdot \pi_{d}}{\pi_{c}+\pi_{d}}
$$

The mean-field equations for $\dot{x_{g}}, \dot{x_{s}}$ and $\dot{y}$, is then gotten as

$$
\begin{aligned}
\dot{x_{g}} & =x(t+1)-x(t) \\
& =\left(x_{g}+\frac{x_{s}^{G}}{\left(x_{s}+y\right)^{G-1}}\right)(1-\xi)^{G}-x_{g} \\
\dot{x_{s}} & =x_{s}(t+1)-x_{s}(t) \\
& =\left(x_{g}+\frac{x_{s}^{G}}{\left(x_{s}+y\right)^{G-1}}\right)\left(1-\xi-(1-\xi)^{G}\right)+x_{s}\left(1-\left(\frac{x_{s}}{x_{s}+y}\right)^{G-1}\right)(1-\xi)+\frac{\xi \cdot \pi_{c}}{\pi_{c}+\pi_{d}}-x_{s} \\
\dot{y} & =y(t+1)-y(t) \\
& =y(1-\xi)+\frac{\xi \cdot \pi_{d}}{\pi_{c}+\pi_{d}}-y,
\end{aligned}
$$

which is Equation 1 .

\section{References}

Aktipis, C. A. (2004). Know when to walk away: Contingent movement and the evolution of cooperation. Journal of Theoretical Biology, 231(2), 249-60

Balliet, D., Wu, J. \& De Dreu, C. K. W. (2014). Ingroup favoritism in cooperation: A meta-analysis. Psychological Bulletin, 140(6), 1556-1581

Bettencourt, B., Charlton, K. \& Kernahan, C. (1997). Numerical representation of groups in cooperative settings: Social orientation effects on ingroup bias. Journal of Experimental Social Psychology, 33(6), 630-659

Carron, A. V. \& Brawley, L. R. (2000). Cohesion: Conceptual and measurement issues. Small Group Research, 31(1), 89-106

Chen, M., Wang, L., Wang, J., Sun, S. \& Xia, C. (2015). Impact of individual response strategy on the spatial public goods game within mobile agents. Applied Mathematics and Computation, 251, 192-202

Chen, X., Fu, F. \& Wang, L. (2009). Social tolerance allows cooperation to prevail in an adaptive environment. Physical Review E, 80, 051104 
Doebeli, M. \& Hauert, C. (2005). Models of cooperation based on the Prisoner's Dilemma and the snowdrift game. Ecology Letters, 8(7), 748-766

Eshel, I. \& Cavalli-Sforza, L. L. (1982). Assortment of encounters and evolution of cooperativeness. Proceedings of the National Academy of Sciences of the United States of America, 79(4), 1331-1335

Evans, C. R. \& Dion, K. L. (1991). Group cohesion and performance: A meta-analysis. Small Group Research, 22(2), $175-186$

Festinger, L. (1950). Informal social communications. Psychological Review, 57(5), 271-282

Fletcher, J. A. \& Doebeli, M. (2006). How altruism evolves: Assortment and synergy. Journal of Evolutionary Biology, 19(5), 1389-1393

Fletcher, J. A. \& Doebeli, M. (2009). A simple and general explanation for the evolution of altruism. Proceedings of the Royal Society B: Biological Sciences, 276(1654), 13-19

Fudenberg, D. \& Maskin, E. (1986). The folk theorem in repeated games with discounting or with incomplete information. Econometrica: Journal of the Econometric Society, 54(3), 533-554

Fujiwara-Greve, T. \& Okuno-Fujiwara, M. (2009). Voluntarily separable repeated Prisoner's Dilemma. The Review of Economic Studies, 76(3), 993-1021

Gully, S. M., Devine, D. J. \& Whitney, D. J. (1995). A meta-analysis of cohesion and performance: Effects of level of analysis and task interdependence. Small Group Research, 26(4), 497-520

Han, T. A., Pereira, L. M. \& Lenaerts, T. (2017). Evolution of commitment and level of participation in public goods games. Autonomous Agents and Multi-Agent Systems, 31(3), 561-583

Hauert, C., De, M. S., Hofbauer, J. \& Sigmund, K. (2002). Replicator dynamics for optional public good games. Journal of Theoretical Biology, 218(2), 187-194

Hayashi, N. (1993). From tit-for-tat to out-for-tat. Sociological Theory and Methods (In Japanese), 8, 19-32

Helbing, D. \& Yu, W. (2009). The outbreak of cooperation among success-driven individuals under noisy conditions. Proceedings of the National Academy of Sciences of the United States of America, 106(10), 3680-3685

Hogg, M. A. (1993). Group cohesiveness: A critical review and some new directions. European Review of Social Psychology, 4(1), 85-111

Hogg, M. A. \& Hains, S. C. (1996). Intergroup relations and group solidarity: Effects of group identification and social beliefs on depersonalized attraction. Journal of Personality and Social Psychology, 70(2), 295-309

Høigaard, R., Säfvenbom, R. \& Tønnessen, F. E. (2006). The relationship between group cohesion, group norms, and perceived social loafing in soccer teams. Small Group Research, 37(3), 217-232

Izquierdo, L. R., Izquierdo, S. S. \& Vega-Redondo, F. (2014). Leave and let leave: A sufficient condition to explain the evolutionary emergence of cooperation. Journal of Economic Dynamics and Control, 46, 91-113

Izquierdo, S. S., Izquierdo, L. R. \& Vega-Redondo, F. (2010). The option to leave: Conditional dissociation in the evolution of cooperation. Journal of Theoretical Biology, 267(1), 76-84

Jaffe, K. (2006). Simulations show that shame drives social cohesion. In J. S. Sichman, H. Coelho \& S. O. Rezende (Eds.), Advances in Artificial Intelligence - IBERAMIA-SBIA 2006, (pp. 88-97). Berlin, Heidelberg: Springer Berlin Heidelberg

Jaffe, K. \& Zaballa, L. (2010). Co-operative punishment cements social cohesion. Journal of Artificial Societies and Social Simulation, 13(3), 4

Joyce, D., Kennison, J., Densmore, O., Guerin, S., Barr, S., Charles, E. \& Thompson, N. S. (2006). My way or the highway: A more naturalistic model of altruism tested in an iterative Prisoners' Dilemma. Journal of Artificial Societies and Social Simulation, 9(2), 4

Li, Y. \& Ye, H. (2018). Effect of the migration mechanism based on risk preference on the evolution of cooperation. Applied Mathematics and Computation, 320, 621-632 
Martinez-Vaquero, L. A., Han, T. A., Pereira, L. M. \& Lenaerts, T. (2015). Apology and forgiveness evolve to resolve failures in cooperative agreements. Scientific Reports, 5(10639)

Mullen, B. \& Copper, C. (1995). The relation between group cohesiveness and performance: An integration. Psychological Bulletin, 115(2), 210-227

Nowak, M. A. (2006). Five rules for the evolution of cooperation. Science, 314(5805), 1560-1563

Paskevich, D. M., Brawley, L. R., Dorsch, K. D. \& Widmeyer, W. N. (1999). Relationship between collective efficacy and team cohesion: Conceptual and measurement issues. Group Dynamics Theory Research \& Practice, 3(3), $210-222$

Perc, M., Jordan, J. J., Rand, D. G., Wang, Z., Boccaletti, S. \& Szolnoki, A. (2017). Statistical physics of human cooperation. Physics Reports, 687, 1-51

Petersen, L.-E., Dietz, J. \& Frey, D. (2004). The effects of intragroup interaction and cohesion on intergroup bias. Group Processes \& Intergroup Relations, 7(2), 107-118

Qu, X., Zhou, C., Cao, Z. \& Yang, X. (2016). Conditional dissociation as a punishment mechanism in the evolution of cooperation. Physica A: Statistical Mechanics and its Applications, 449, 215 - 223

Rand, D. G., Tarnita, C. E., Ohtsuki, H. \& Nowak, M. A. (2013). Evolution of fairness in the one-shot anonymous ultimatum game. Proceedings of the National Academy of Sciences of the United States of America, 110(7), $2581-2586$

Rob, R. \& Yang, H. (2010). Long-term relationships as safeguards. Economic Theory, 43(2), 143-166

Rong, Z., Wu, Z.-X. \& Chen, G. (2013). Coevolution of strategy-selection time scale and cooperation in spatial Prisoner's Dilemma game. EPL (Europhysics Letters), 102(6), 68005

Rubinstein, A. (1979). Equilibrium in supergames with the overtaking criterion. Journal of Economic Theory, 21(1), 1-9

Sasaki, T., Okada, I. \& Unemi, T. (2007). Probabilistic participation in public goods games. Proceedings of the Royal Society B. Biological Sciences, 274(1625), 2639-2642

Schuessler, R. (1989). Exit threats and cooperation under anonymity. Journal of Conflict Resolution, 33(4), 728749

Semmann, D., Krambeck, H.-J. \& Milinski, M. (2003). Volunteering leads to rock-paper-scissors dynamics in a public goods game. Nature, 425,390

Szabó, G. \& Fath, G. (2007). Evolutionary games on graphs. Physics Reports, 446(4), 97-216

Szolnoki, A. \& Chen, X. (2015). Benefits of tolerance in public goods games. Physical Review E, 92, 042813

Vainstein, M. H., Silva, A. T. \& J, J. (2007). Does mobility decrease cooperation? Journal of Theoretical Biology, 244(4), 722-8

Van Segbroeck, S., Pacheco, J. M., Lenaerts, T. \& Santos, F. C. (2012). Emergence of fairness in repeated group interactions. Physical Review Letters, 108, 158104

Van Veelen, M., García, J., Rand, D. G. \& Nowak, M. A. (2012). Direct reciprocity in structured populations. Proceedings of the National Academy of Sciences of the United States of America, 109(25), 9929-9934

Weibull, J. W. (1997). Evolutionary Game Theory. Cambridge, MA: MIT Press

Wubs, M., Bshary, R. \& Lehmann, L. (2016). Coevolution between positive reciprocity, punishment, and partner switching in repeated interactions. Proceedings of the Royal Society B: Biological Sciences, 283(1832), 20160488

Zisis, I., Di Guida, S., Han, T., Kirchsteiger, G. \& Lenaerts, T. (2015). Generosity motivated by acceptanceevolutionary analysis of an anticipation game. Scientific Reports, 5, 18076 